\title{
Effect of Fatigue on the Proprioception Acuity of Quadriceps Muscle after Inducing Fatigue and Followed By Local Cooling in the Same Muscle
}

\author{
Ravindra Sharma
}

\begin{abstract}
Aim: To study the effect of fatigue on the proprioception acuity of quadriceps muscle after inducing fatigue and followed by local cooling. Method: The study was conducted on 30 males (age $22.53 \pm 2.12$ years, BMI $22.27 \pm 1.52$ ). Pre test and post test responses regarding the weight discrimination task were recorded both before and after inducing fatigue with local cooling. Results: Mean values of correct responses for weight discrimination task $(60 \mathrm{gm}, 120 \mathrm{gm}, 180 \mathrm{gm}$ and $240 \mathrm{gm})$ for pre test were $0.83 \pm 0.379,0.73$ $\pm 0.45,0.7 \pm 0.46,0.83 \pm 0.379$, after fatigue were $0.5 \pm 0.509,0.5 \pm 0.509,0.5 \pm 0.509$, $0.4 \pm 0.498$ and fatigue with cooling were $0.23 \pm 0.43,0.3 \pm 0.46,0.3 \pm 0.46,0.13 \pm 0.346$ respectively. Conclusion: It concluded that the proprioceptive acuity is reduced after fatigue and cooling further reduces the acuity of a fatigued muscle.
\end{abstract}

Ravindra Sharma

Professor

Prem Physiotherapy and Rehabilitation College

Panipat, Haryana, India

E-mail: goldravi76@gmail.com
Key Words: Joint position sense, Muscle strength, Weight discrimination, Cryotherapy

DOI: $10.18376 / j e s p / 2017 / v 13 / \mathbf{i} 2 / 111286$

\section{Introduction}

Human co-ordination skill and capability to accomplish targets, as simple yet as complex, as activities of daily living are possible by a synchronized neuromuscular activity central to which are the sensations eminating from various structures (Bosco and Poppele 2001). Of all these, proprioception or joint position sense seems to play the most important role in joint function by which the body can vary muscle contraction in immediate response to incoming information regarding external forces. Numerous studies have found that intact joint position was a prerequisite for normal muscle co-ordination and a proper feedback to the central nervous system. They also concluded that, in presence of muscular fatigue there is a poprioceptive deficit in the joints (Brown JP et al 2002, Carpenter JE et al 1998, Lee HM et al 2003). The human proprioceptive system not only has the ability to sense joint position but also has the ability to sense movement and to perceive weight and force from both central and peripheral signals. The signals related to the muscle length - tension changes are carried via the Group Ia, Ib and II of the Type A nerve fibres from the primary nerve endings in the muscle spindle and the Golgi tendon organs in the muscle tendon till the spinal cord via the Dorsal root ganglia and terminate in the Clarke's column in Rexed's lamina VII of the lumbar and thoracic spinal cord segments. The ability of weight discrimination is inherent to the muscles and that the same is more accurate when the objects are lifted actively rather than being passively applied on the skin. Muscle fatigue is defined as a transient decrease in maximal voluntary performance capacity of a muscle. The metabolic changes that accompany fatigue directly affect the contractile machinery and activate afferents, which may induce a reduction in force. Factors such as depletion of ATP levels, depletion of neurotransmitter reserves, increased concentration 
of inorganic phosphates $(\mathrm{Pi})$ at the cross-bridges level, increased concentration of $\mathrm{H}^{+}$ions, decreased concentration of intracellular potassium ion $(\mathrm{K}+)$, increase in extra cellular potassium ion $(\mathrm{K}+)$ and increased threshold of synaptic or motor end plate receptors lead to the decrease in muscle performance capacity (Kirkendall DT, 1990). Various studies have pointed out that, muscular fatigue produces a great deal of proprioceptive deficits regarding movement sense and postural control along with passive repositioning of the joints (Forestier $\mathrm{N}$ et al., 2002, Gribble PA and Hertel J, 2004, Johnson RB III et al., 1998). Cryotherapy, the application of cold or ice for the treatment of acute or overuse injury, was first mentioned by Hippocrates around $400 \mathrm{BC}$ and has been widely used ever since. It is cheap, easily available and effective. Cryotherapy is regarded as the preferred choice for treating on-field sporting injuries and henceforth hastens the return for athletic participation (Berg U and Ekblom B 1979). Superficial application of ice results in decreased intramuscular temperature and stimulation of the cutaneous receptors leads to excitation of the sympathetic adrenergic fibres to constrict local arterioles and venoules. Vasoconstriction reduces the swelling associated with bleeding and inflammation. It leads to a decreased rate of metabolism and production of metabolites, as well as decreased oxygen requirements, therefore limiting the extension of the injury to uninjured tissues (Weimar W and Campbell B 2004).

\section{Materials and Methods}

In the pre-assessment the following recordings were carried out in all the subjects age, height (in meters), weight (in kilograms), skin temperature at the thigh (in ${ }^{\circ} \mathrm{C}$ ), IRM, $75 \%$ of IRM, body mass index (BMI). Every subject's Quadriceps femoris muscle group whose right side was dominant, were chosen for the weight discrimination task following fatigue and application of local cooling. Each subject was blindfolded and positioned in high sitting with their thighs well supported on a leg exerciser in which the right side femoral epicondyle was aligned with the axis of the lever arm of the leg exerciser. In weight discrimination task subjects were instructed that they were free to choose any movement ranges from (90 degrees to 180 degrees), speed and mode of contraction (concentric or eccentric) they deemed appropriate to estimate the weights. The subject were asked to do four active lifts of randomly added small metallic weights $(0.06 \mathrm{~kg}$, $0.12 \mathrm{~kg}, 0.18 \mathrm{~kg}, 0.24 \mathrm{~kg}$ ) to the lever arm of the leg exercise unit and their responses will be recorded. (minimum of $75 \%$ correct response was required) on the data collection sheet. For inducing fatigue, IRM of each subject was calculated by the formula,

Maximum weight (kgs)

$\mathrm{IRM}=$

$1.0278-0.0278(\mathrm{~N})$

Where,

$\mathrm{N}$ number of repetitions done (should be less than 10 repetitions) and, 1.0278 and 0.0278 are constant values (Bryzcki M 1993). Immediately after the completion of post fatigue weight discrimination task, each subject was made to lie supine with the anterior aspect of the right thigh exposed excluding the most distal (patellar) and proximal (groin) areas. Cooling in the form of cold packs (Cree CD, 1999) was applied over the anterior two-third aspect of the right thigh to obtain effective cooling of the Quadriceps muscle belly. After removal of the cold packs the skin was inspected. Each subject reported diminished sensation and a decreased response to pin prick test in the cooled area. Each subject was again positioned on the leg extension exerciser within 2-3 minutes and the weight discrimination task was performed again. To avoid any pressure cues the lever was pulled away from the subject's leg during loading and unloading of weights. 
Data was analysed by using Software Analyze - IT for MS-Excel and manually Chi Square was used to compare the correct responses. One way ANOVA was used to compare the mean scores of different stages.

\section{Results and Discussion}

\section{Results}

Using Chi-Square test on the data, the results were found to be significant at $p<0.001$, for analysis of changes in the proprioceptive acuity after fatigue and application of local cooling. This suggests that experimental hypothesis has been supported for $0.06 \mathrm{kgs}, 0.12 \mathrm{kgs}, 0.18 \mathrm{kgs}$, and $0.24 \mathrm{kgs}$ of weight taken. Thus, the null hypothesis therefore is rejected. Further, by using the One-way ANOVA for related subject samples on data $(\mathrm{F}=21.9 ; \mathrm{N}=30)$, it was found that the results are highly significant at $p<0.01$. This suggested that with increased weight there was a significant proprioceptive loss. Hence, at $0.24 \mathrm{kgs}$, loss of proprioceptive acuity was maximum.

Table 1. Descriptive statistics of the subjects

\begin{tabular}{|l|c|c|}
\hline Variables & Mean & Standard Deviation \\
\hline Age (years) & 22.53 & 2.12 \\
\hline BMI & 22.27 & 1.5 \\
\hline 1 RM & 43.41 & 5.48 \\
\hline $75 \%$ of 1RM & 32.83 & 3.70 \\
\hline Skin temperature before & 27.8 & 0.81 \\
\hline Skin temperature after & 14.73 & 1.01 \\
\hline
\end{tabular}

Table 2. Comparison of Mean Values

\begin{tabular}{|l|c|c|}
\hline Weights & Chi square value & F value \\
\hline 60 gms & 20.375 & 13.84 \\
\hline 120gms & 7.464 & 6.24 \\
\hline 180gms & 11.332 & 5.19 \\
\hline $240 g m s$ & 30.131 & 21.90 \\
\hline
\end{tabular}

Table 1 shows mean age, 1RM, $75 \%$ of $1 \mathrm{RM}$, BMI, surface temperature before and after cooling. Table 2 shows comparison of mean values at different stages for $60 \mathrm{gm}, 120 \mathrm{gm}, 180$ $\mathrm{gm}$ and $240 \mathrm{gm}$. It was observed that there were changes in the proprioceptive acuity after fatigue and application of local cooling.

\section{Discussion}

In accordance with the previous studies, this study has shown that the proprioceptive acuity in the quadriceps muscle, as reflected in the ability to differences in weight, was decreased after inducing fatigue followed by application of local cooling which is in general agreement with the studies done by Johnson et al on fatigue and Cree et al on cryotherapy. The data of this study does not support the findings that proprioceptive acuity is preserved after local cooling and fatigue respectively. Fatigue increases the threshold of muscle spindle discharge, which disrupts the afferent feedback subsequently altering the joint awareness for normal 
proprioception cutaneous input and the sensitive of the muscle spindle are the most important requirements. Cooling over a nerve fibre area causes a decrease in the rate of impulse transmission along the nerve fibre, regardless of the type or function of the fibre. We used conventional methods for inducing fatigue and in applying local cooling. Fatigue was induced by making the subject lift $75 \%$ of his 1 Repetition Maxirnum (RM) for 20 times. Local cooling was applied in the form of ice packs and the surface tissue temperature was recorded pre and post cooling. Our surface readings were similar to those who have reported significant reductions in intramuscular temperatures. Thus, we can reasonably assume that our cooling method was effective in decreasing muscle temperatures. The degree of cooling achieved in each individual may have been different due to thickening of the underlying adipose tissue. Nevertheless all participants reported diminished sensation in the cooled area (response to pinprick) after the exhibiting the usual usual objective signs of tissue cooling such as intense skin redness. After analyzing the data the present findings were highly significant $(\mathrm{p}<0.0101)$. Since there is a rush of sporting activities it places tremendous psychological, mechanical \& physiological demands upon the nervous system of an athlete. Since, proprioception involves both neural as well as muscular components, it is probable that effect of intra-muscular environment like fatigue and reduced temperature could affect the accuracy of this sensation.

\section{Conclusion}

It concluded that the proprioceptive acuity is reduced after fatigue and cooling further reduces the acuity of a fatigued muscle.

\section{Acknowledgement}

Thank to all the subjects who voluntarily participated in this study.

\section{References}

Berg U and Ekblom B. 1979. Influence of muscle temperature on maximal strength and power output in human skeletal muscle. Acta Physiol Scand 107: 33-37.

Bosco G and Poppele RE. 2001. Proprioception from a spinocerebellar perspective. Physiol Rev 81(2): 539-568

Brown JP and Bowyer GW. 2002. Effect of fatigue on ankle stability and proprioception in university sports people.Br J Sports Med 36: 310-312.

Bryzcki M. 1993. Strength testing - predicting a one - rep max from reps to fatigue. Journal of Physical Education, Recreation and Dance 64: 88-90

Carpenter JE, Blaiser RB, Pellizzon GG. 1998. Effect of muscle fatigue on shoulder joint position sense. Am J Sports Med 26: 262-265.

Cree CD. 1999. Frostbite at gym: it's not the ice but the temperature that matters. Br J Sports Med 33: 435436.

Forestier N, Teasdale N, Nougier V. 2002. Alteration of the position sense at the ankle induced by the muscular fatigue in humans. Med and Science in Sports and Exercise 34(1):117-122.

Gribble PA and Hertel J.2004. Effect of lower extremity fatigue on postural control. Arch Phys Med Rehabil 85(4): 589-592.

Johnson RB III, Howard ME, Cawley PW, Losse GM. 1998. Effect of lower extremity fatigue on motor control performance. Med and Science in Sports and Exercise 30(12):1703-1707.

Kirkendall DT. 1990. Mechanism of peripheral fatigue. Med and Science in Sports and Exercise 22(4):444-449.

Lee HM, Liau JJ, Cheng CK, Tan CM, Shin JT. 2003. Evaluation of shoulder proprioception following muscle fatigue. Clin Biomech 18(9):843-847.

Weimar W, Campbell B.2004. The influence of ankle cryotherapy on unilateral static balance. Med and Science in Sports and Exercise 36(5): 187-190. 